\title{
Study on the Experiments and the Numerical Analysis of Exchange Flow Behavior in the Unstably Stratified Field
}

\author{
Motoo Fumizawa, Yoshiharu Saito, Naoya Uchiyama, Takahiro Nakayama \\ Department of Mechanical Engineering, Shonan Institute of Technology Fujisawa, Kanagawa, Japan \\ Email address: \\ fumizawa@mech.shonan-it.ac.jp (M. Fumizawa), sutere0port96@gmail.com (Y. Saito), psn-man.lv.030@softbank.ne.jp (N. Uchiyama), \\ 2991taka@gmail.com (T. Nakayama)
}

\section{To cite this article:}

Motoo Fumizawa, Yoshiharu Saito, Naoya Uchiyama, Takahiro Nakayama. Study on the Experiments and the Numerical Analysis of Exchange Flow Behavior in the Unstably Stratified Field. International Journal of Energy and Power Engineering.

Vol. 4, No. 3, 2015, pp. 178-183. doi: 10.11648/j.ijepe.20150403.16

\begin{abstract}
In the flow mechanism of unstably stratified field, occurs after Rayleigh-Taylor instability. Buoyancy-driven exchange flows were investigated the helium-air flow in the vertical narrow pathway between upper air chamber and lower helium chamber. Exchange flows may occur following the opening of a window for ventilation, when fire breaks out in a room, as well as when a pipe ruptures in a high temperature gas-cooled nuclear reactor. The numerical analysis and experiment in this paper was carried out in a test chamber filled with helium and the flow was visualized using the smoke wire method. The flow behavior was recorded by a high-speed camera combined with a computer system. The image of the flow was transferred to digital data, and the flow velocity was measured by PTV and PIV software. The mass fraction in the test chamber was measured using electronic balance. The detected data was arranged by the densimetric Froude number of the exchange flow rate derived from the dimensional analysis. A method of mass increment was developed and applied to measure the exchange flow rate. As the result, it is revealed that three dimensional structure of counter current exchange flow in the narrow flow path such as rotation and circulation flows by the optical system, mass inclement method and numerical analysis of moving particle method as well as HSMAC method.
\end{abstract}

Keywords: Buoyancy, Exchange Flow, Helium, Moving Particle Method, Clockwise Flow

\section{Introduction}

In the flow mechanism of unstably stratified field, occurs after Rayleigh-Taylor instability. Buoyancy-driven exchange flows were investigated the helium-air flow in the vertical narrow pathway between upper air chamber and lower helium chamber. Exchange flows may occur following the opening of a window for ventilation, the outbreak of fire in a room or over an escalator in an underground shopping center, as well as when a pipe ruptures in a modular high temperature gas-cooled nuclear reactor. The fuel loading pipe is located in an inclined position in a pebble bed reactor such as the Modular reactor [1,2,3] and AVR [4].

In safety studies of High Temperature Gas-Cooled Reactor (HTGR), the failure of a standpipe at the top of the reactor vessel or a fuel loading pipe may be one of the most critical design-based accidents. Once the pipe ruptures, helium immediately rushes up through the breach. Once the pressure between the inside and outside of the pressure vessel has balanced, helium flows upward and air flows downward through the breach into the pressure vessel. This means that buoyancy-driven exchange flow occurs through the breach, caused by the density difference of the gases in the unstably stratified field. Since an air stream corrodes graphite structures in the reactor, it is important to evaluate and reduce the air ingress flow rate when a standpipe rupture occurs.

Studies have been performed on the exchange flow of two fluids with different densities through vertical short tubes. Epstein[5] studied the exchange flow of water and brine through various vertical tubes, experimentally and theoretically. Mercer et al.[6] experimentally studied an exchange flow through inclined tubes with water and brine. The latter experiments were carried out in the range of 3.5 $<\mathrm{L} / \mathrm{D}<18$ and $0 \mathrm{deg}<\theta<90 \mathrm{deg}$, and indicated that the length-to-diameter ratio $\mathrm{L} / \mathrm{D}$, and the inclination angle $\theta$ of the tube are the important parameters for the exchange flow 
rate. Most of these studies were performed on the exchange flow using a relatively small difference in the densities of the two fluids (up to 10 per cent). However, in the case of a HTGR standpipe rupture, the density of the outside gas is at least three times larger than that of the gas inside the pressure vessel. Few studies have been performed so far using such a large density difference. Kang et al.[7] studied experimentally the exchange flow through a round tube with a partition plate. Although one may assume that the partitioned plate, a kind of obstacle in the tube, would reduce the exchange flow rate, Kang found that the exchange flow rate was increased by the partition plate because of separation of the upward and downward flows.

The main objectives of the present study are to investigate the behavior of the exchange flow in the vertical narrow pathway then to evaluate the exchange flow rate using mass increment with the helium-air system. The following methods are applied the present study.

(1) Optical system of the Mach-Zehnder interferometer

(2) Numerical analysis

(3) Mass increment method

\section{Optical System of Mach- Zehnder Interferometer}

\subsection{Experimental Apparatus and Procedure}

The optical system of the Mach-Zehnder interferometer, MZC-60S to visualize the exchange flow is shown in Figure 1. After being rejoined behind the splitter, the test and reference laser beams interfere, and the pattern of interference fringe appears on the screen. If the density of the test section is homogeneous, the interference fringes are parallel and equidistant [8,9]. If the density is not homogeneous, the interference fringes are curved. Inhomogeneity in the test section produces a certain disturbance of the non-flow fringe pattern. The digital camera and high-speed camera using a D-file can be attached to the interferometer.

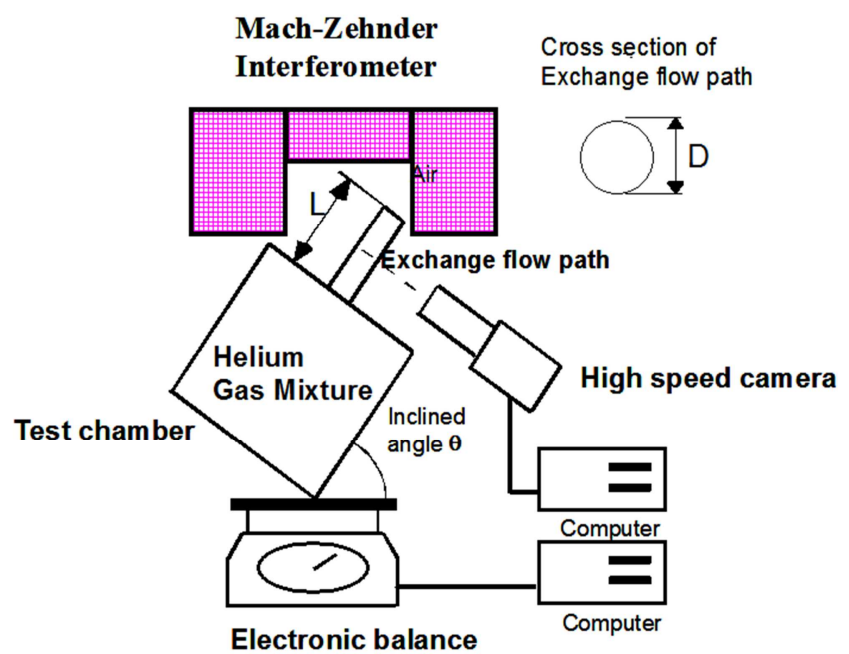

Fig. 1. Experimental apparatus of optical system of Mach-Zehnder.

\subsection{Results of Optical System}

To investigate the flow pattern, the exchange flows are visualized by the Mach-Zehnder interferometer. The photo in Figure 2 shows the typical interferogram of the fringes for the vertical narrow tube $(\mathrm{L} / \mathrm{D}=5)$. The upward helium plume and the downward air plume break intermittently through the passage and swing from left to right in the lateral direction. The period of the swing is $\sim 2$ s.It is clearly observed that the up flow of helium and the down flow of air do not take place smoothly, because they interact strongly with each other. The flow is fluctuated and unstable.

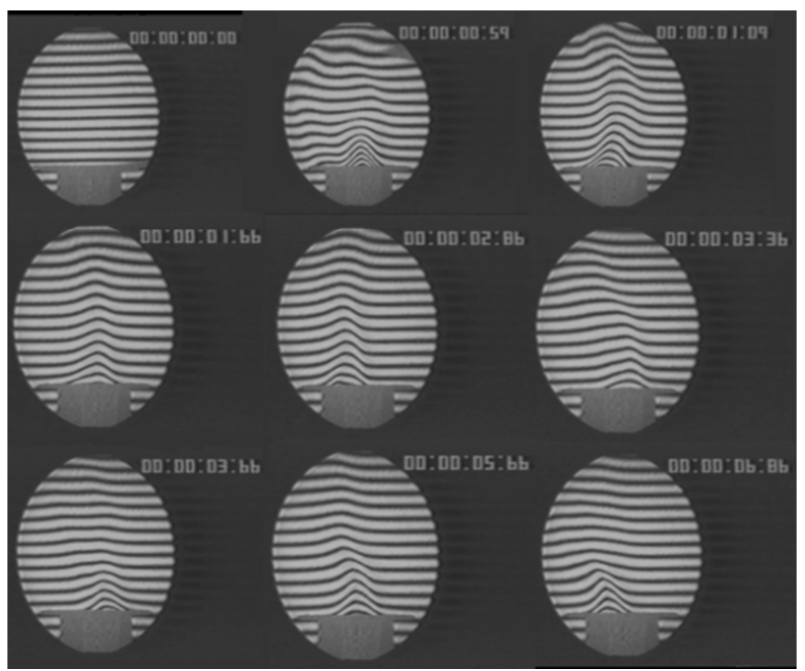

Fig. 2. Visualization result of Mach-Zehnder method upper part of vertical narrow tube.

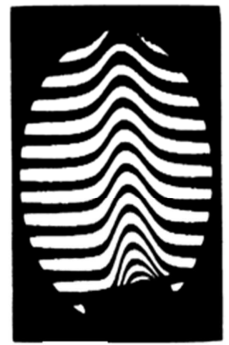

(a) $\theta=15^{\circ}$

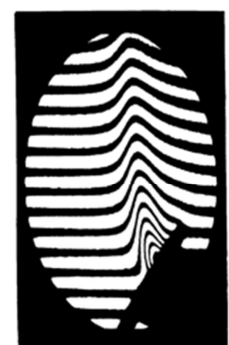

(d) $\theta=60^{\circ}$

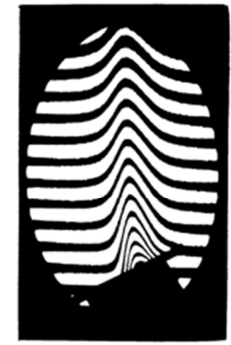

(b) $\theta=30^{\circ}$

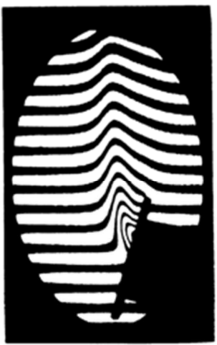

(e) $\theta=75^{\circ}$

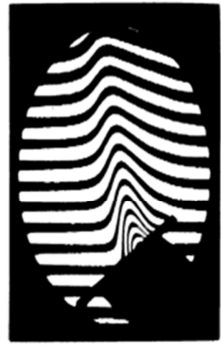

(c) $\theta=45^{\circ}$

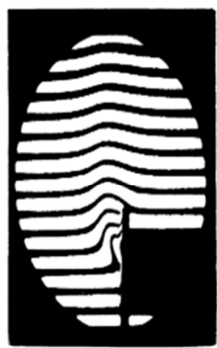

(f) $\theta=90^{\circ}$
Fig. 3. Typical interference fringes for an inclined narrow tube $(L / D=5)$.

Figure 3 shows the typical interference fringes for an inclined long tube $(\mathrm{L} / \mathrm{D}=5)$. The curved interference fringes indicate that the lighter helium flows in the upper passage of the tube. The straight fringes indicate that the heavier air 
flows in the bottom of the tube. It is clearly visualized that the exchange flows take place smoothly and in a stable manner in the separated passages of the tube. This leads to less resistance for the exchange flow in the inclined tubes compared to the vertical ones. In the case of a $30 \mathrm{deg}$. angle, the curvature of the interference fringes is larger than that at other angles, indicating that the exchange flow rate and the densimetric Froude number are the largest at $30 \mathrm{deg}$.

\section{Numerical Analysis}

\subsection{Moving Particle Method}

Figure 4 and 5 show cross sectional view and bird-eye view of numerical boundary conditions of moving particle method of 3D coordinate, respectively. Table 1 shows the calculation condition of moving particle method. Lagrange method is adopted calculation program of moving particle method. Therefore, the numbers of particles are around two million. The calculation program code is adopted Particleworks3.01 and possessor is composed of TESLA C2070 [10].

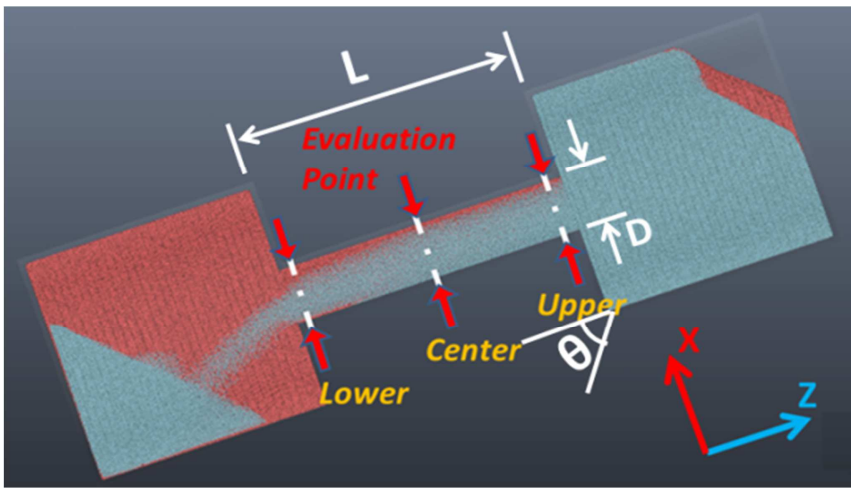

Fig. 4. Cross sectional view of numerical boundary conditions of moving particle method.

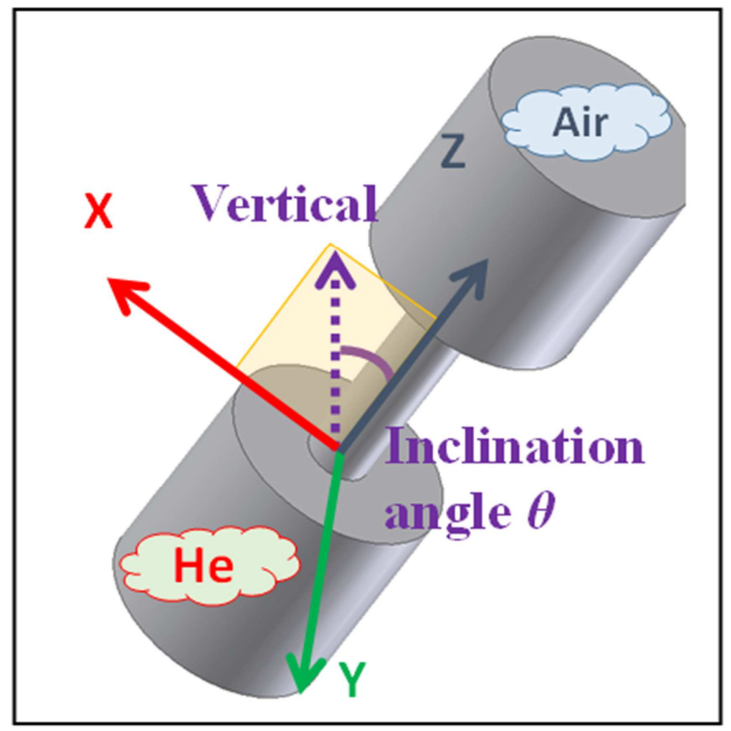

Fig. 5. Bird -eye view of numerical boundary conditions of moving particle method.
Table 1. Calculation condition of moving particle method.

\begin{tabular}{ll}
\hline Number of particle $\boldsymbol{N}$ & $\mathbf{1}$ 2million \\
\hline Inclination angle $\theta$ & $0^{\circ}, 15^{\circ}, 30^{\circ}, 45^{\circ}, 90^{\circ}$ \\
Particle diameter $a$ & $0.815 \sim 1.0 \mathrm{~mm}$ \\
Flow path length $L$ & $100 \mathrm{~mm}$ \\
Diameter flow path $d$ & $20 \mathrm{~mm}$ \\
Container size $\mathrm{h}=D$ & $80 \mathrm{~mm}, 110 \mathrm{~mm}$ \\
\hline
\end{tabular}

Figure 6 shows unstably and asymmetric flow patterns became dominant in the vertical narrow pipe. It is interesting that the horizontal cut view, in the figure, reveals that the clockwise upward flow was observed, which means spiral upward flow pattern. Figure 7 shows that the upward plume behaves like the snake movement in the upper chamber. Numerical calculation of moving particle method predicts that the clockwise movement of the plume and snake behavior of plume and the behavior is consistent with the result that Mach-Zehnder method shows random behavior of the plume from left to right.

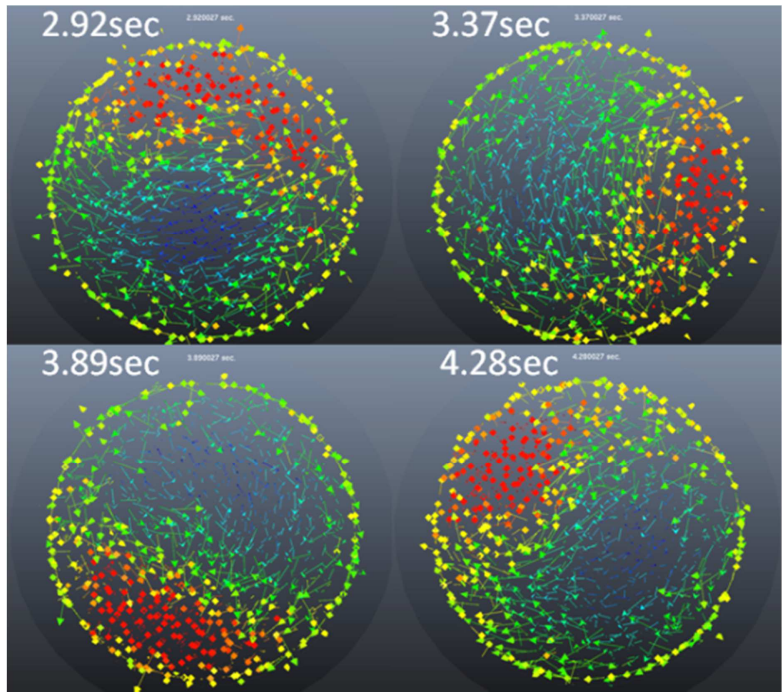

Fig. 6. Horizontal view of exchange flow in the narrow tube.

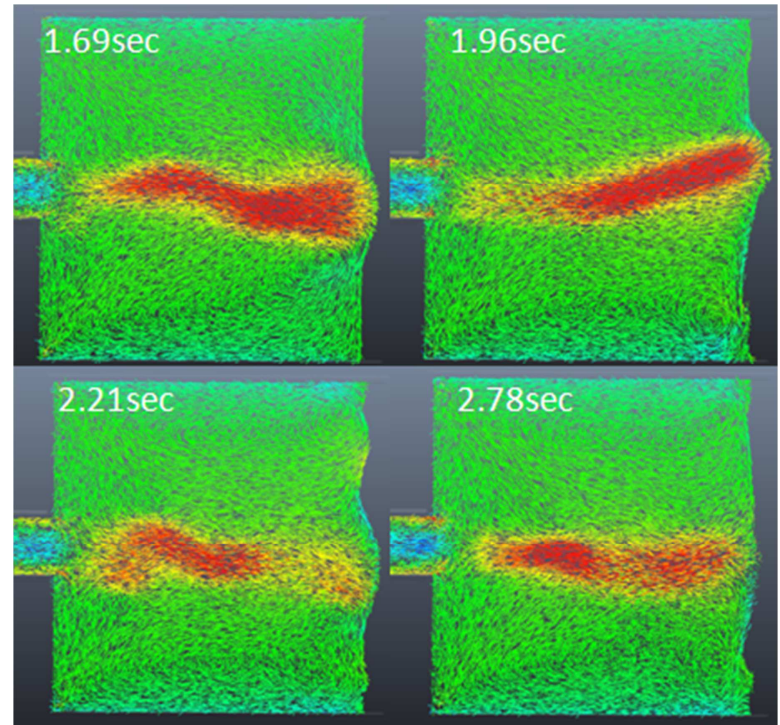

Fig. 7. Vertical view of light plume behavior upper chamber on the narrow tube. 


\subsection{HSMAC Method}

Two dimensional unsteady code of HSMAC method is adopted to the buoyancy-driven exchange flow system[2]. In the code, FORTRAN program is described basic equation of mass, momentum and energy. Analysis coordinate is shown in Figure 8. The left part is test chamber filled with helium gas and the right part is outside region filled with air. Typical calculation result is shown in Figure 9, where is the narrow channel, between the left and right. The exchange flow occurs with vortex in the narrow channel. Therefore, the center flow rate $\mathrm{Q}_{1}$ is larger than the right edge flow rate $\mathrm{Q}_{2}$, as shown in Figure 10.

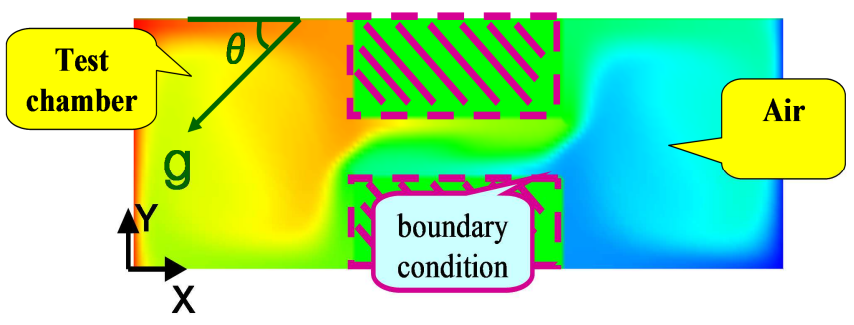

Fig. 8. Analytical coordinate and conditions.

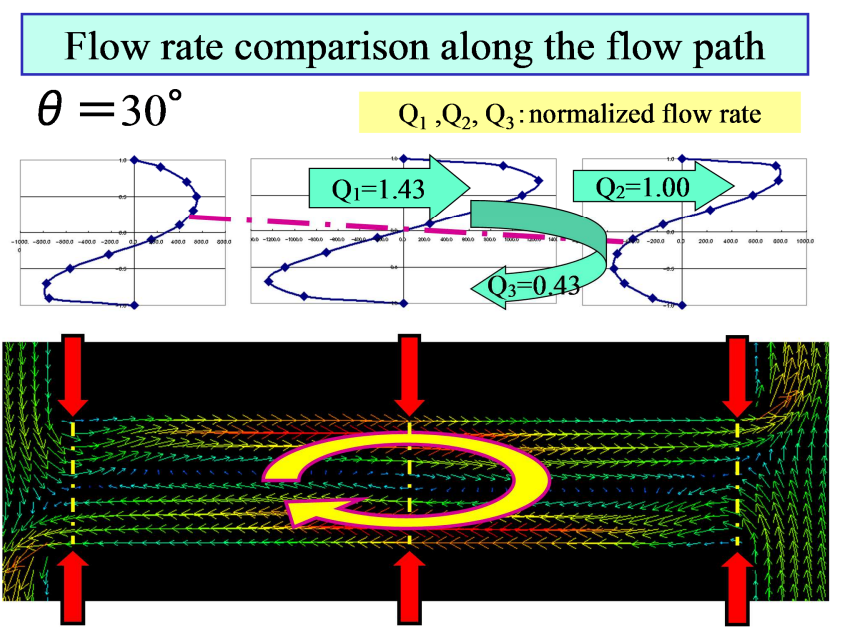

Fig. 9. Analytical result of narrow flow path of Exchange flow.

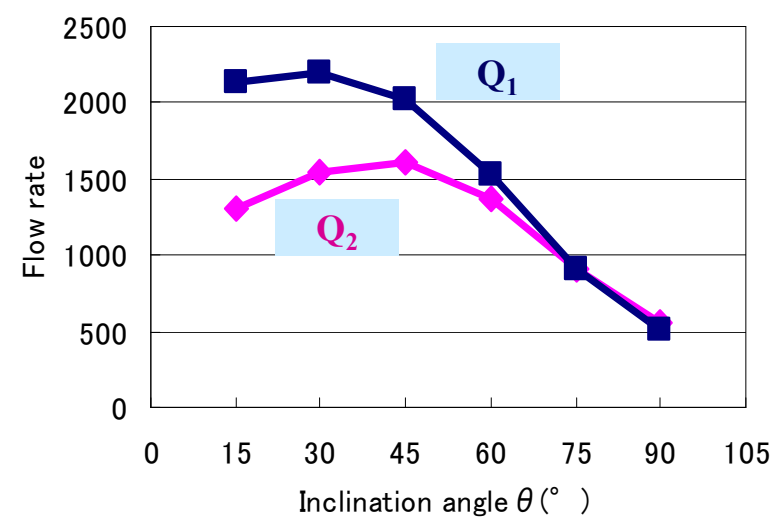

The Flow rate Q1 is higher than Q2

Fig. 10. Relation of Exchange flow rate and inclination angle of analytical results.

\section{Method of Mass Increment}

\subsection{Experimental Apparatus and Procedure}

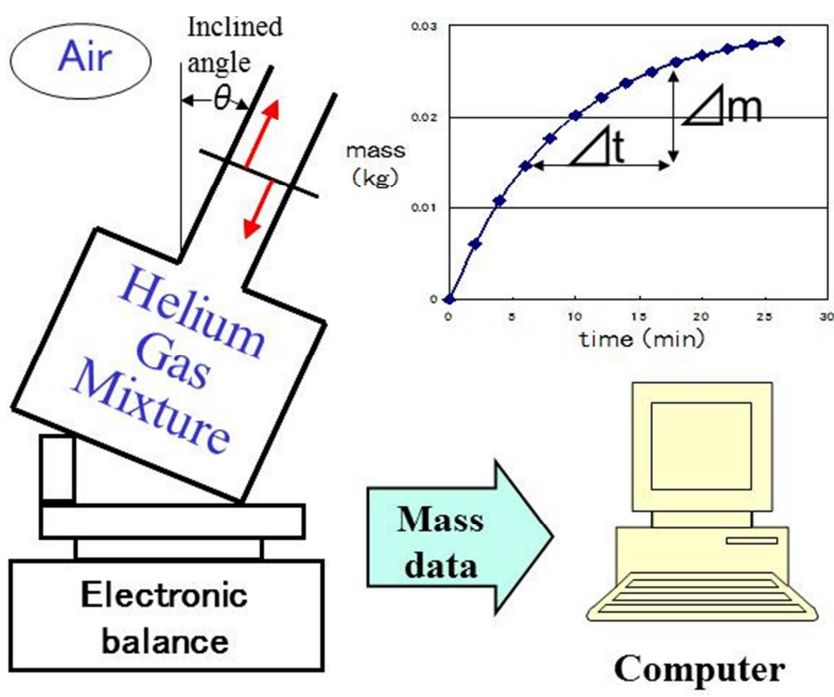

Fig. 11. Sketch of the mass increment apparatus.

The mass increment method was used for the investigations. Figure 11 shows a rough sketch of the apparatus. It consists of a test chamber, an electronic balance and a personal computer for data acquisition. The experimental procedure are as follows. Air enters the test chamber and the mass of the gas mixture in the test chamber increases. The mass increment $\Delta \mathrm{m}$ is automatically measured by the high accurate electronic balance. The density increment of the gas mixture $\Delta \rho_{\mathrm{L}}=\Delta \mathrm{m} / \mathrm{V}$ is calculated From mass increment data,. The density increment signifies the difference between densities of the gas mixture from the density of pure helium in the test chamber. Subsequently, the volumetric exchange flow rate is evaluated using the following equation:

$$
Q=\frac{V}{\rho_{H}-\rho_{L}} \cdot \frac{d\left(\Delta \rho_{L}\right)}{d t}
$$

The densimetric Froude number is defined by the following equation derived from the dimensional analysis suggested by Keulegan [11]:

$$
F r=\frac{Q}{A} \sqrt{\frac{\rho}{g D \Delta \rho}}
$$

In the above equations, $\mathrm{V}$ is the volume of the test chamber, $\rho_{\mathrm{H}}$ the density of air, $\rho_{\mathrm{L}}$ the density of the gas mixture in the test chamber, $\triangle \rho_{\mathrm{L}}\left(=\rho_{\mathrm{H}}-\rho_{\mathrm{He}}\right)=$ the density increment of the gas mixture, $\mathrm{t}$ the elapsed time, $\mathrm{U}(=\mathrm{Q} / \mathrm{A})$ the exchange-velocity, $\rho\left(=\rho_{\mathrm{H}}+\rho_{\mathrm{L}}\right) / 2$, D the diameter and $g$ the acceleration of gravity. The experiments are performed under atmospheric pressure and room temperature using the vertical and inclined round tubes, and using a vertical annular tube. The density of the gas mixture is close to that of helium in the present experiment. The sizes of the tubes are as follows. 
The diameter of the round tube $\mathrm{D}$ is $20 \mathrm{~mm}$, which is much smaller than that of the test chamber. The inclination angle $\theta$ ranges from 15 to $90 \mathrm{deg}$ and the height $\mathrm{L}$ ranges from 0.5 to $200 \mathrm{~mm}$.

\subsection{Comparison between Two Experimental Methods}

It is already known that the densimetric Froude number is regarded as constant within a time duration when the gas in the upward flow is assumed to be helium [12]. Figure 12 shows the relationship between $\mathrm{Fr}$ and inclination angle $\theta$ with $\mathrm{L} / \mathrm{D}$ as a parameter. For inclined tubes, Fr is larger than that for vertical tubes. (i.e. $\mathrm{L} / \mathrm{D}=10$ ). The densimetric Froude number reaches the maximum at $60 \mathrm{deg}$ for the orifice and $30 \mathrm{deg}$ for the long tube. It is found that the angle for the maximum Fr decreases with the increase of $\mathrm{L} / \mathrm{D}$ in the helium-air system.

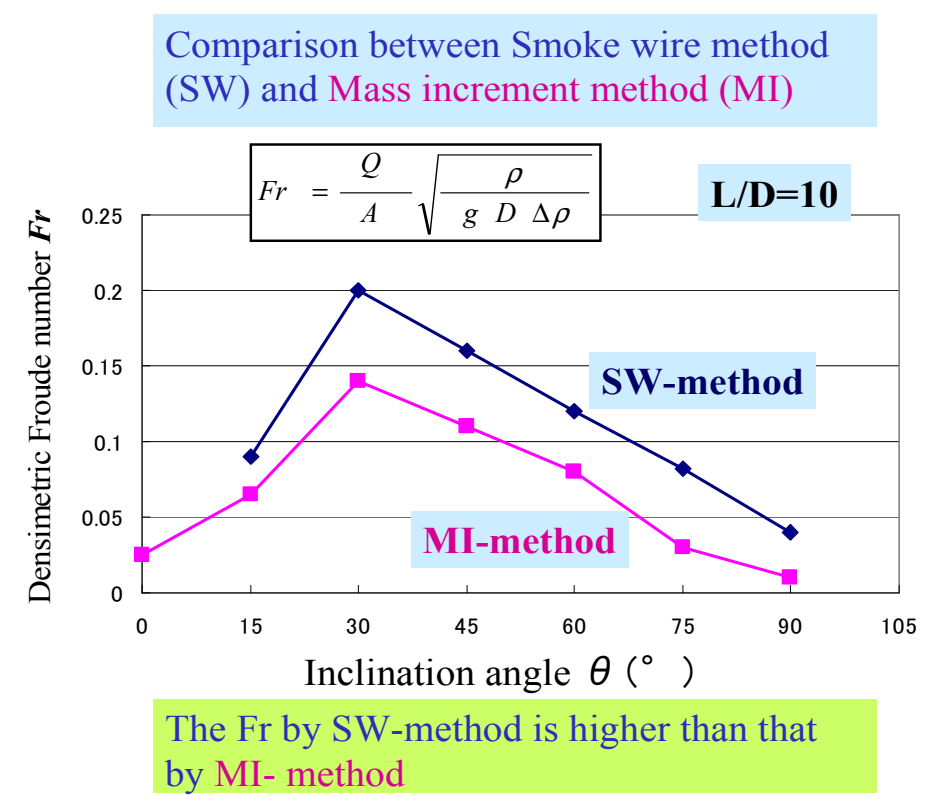

Fig. 12. Relation of Densimetric Froude number and inclination angle in both experiments.

\section{Conclusion}

It is revealed that three dimensional structure of counter current exchange flow in the narrow flow path such as rotation and circulation flows by the optical system, mass inclement method and numerical analysis of moving particle method as well as HSMAC method.

(1) Numerical calculation of moving particle method predicts the clockwise movement of the plume behavior flow occurs in the narrow flow path

(2) Numerical calculation of HSMAC method predicts the circulation flow of the tube length direction occurs in the narrow flow path.

(3) Numerical calculation of moving particle method predicts snake behavior of plume and the behavior is consistent with the result that Mach-Zehnder method shows random behavior of the plume from left to right.

(4) (4) Densimetric Froude number Fr by SW-method is higher than that by MI- method.

\section{Acknowledgements}

The authors are deeply indebted to Mr. Shuhei Ohkawa, working Sanwa-koki Co. Ltd., for his unfailing interest and helpful input to this study.

\section{References}

[1] Fumizawa,M.; Nuclear Reactors (ISBN 978-953-51-0018-8), Edited by Amir Zacarias Mesquita, InTech, pp.47-56 (2012)

[2] Fumizawa,M.; Proc. HT2005 ASME Summer Heat Transfer Conference, HT2005 -72131, Track 1-7-1, pp.1-7 (2005).

[3] Fumizawa,M.;Nuclear Reactors (ISBN 978-953-51-0967-9), Edited by Amir Zacarias Mesquita, InTech, pp.177-191 (2013)

[4] M.M.El-Wakil, Nuclear Energy Conversion, Thomas Y. Crowell Company Inc., USA (1982)

[5] Epstein,M., Trans. ASME J. Heat Transfer, pp.885 -893 (1988)

[6] Mercer, A. and Thompson.H., J. Br. Nucl. Energy Soc., 14, pp.327-340 (1975),

[7] Kang,T. et al., NURETH-5, pp.541-546 (1992)

[8] Merzkirch.W., "Flow Visualization", Academic Press (1974)

[9] Feng,J. et al., Chemical Engineering Journal, Volume 86, pp.243-250 (2002)

[10] Prometech software web-site, http://www.prometech.co.jp/ (2015)

[11] Keulegan, G. H., U. S. N. B. S. Report 5831 (1958) 
[12] Fumizawa,M. et. al., J. At. Energy Soc. Japan, Vol.31, pp.1127-1128 (1989) 